\title{
DEFORMATIONS OF GENERALIZED COMPLETE INTERSECTIONS
}

\author{
R. MANDELBAUM AND M. SCHAPS
}

\begin{abstract}
The concept of a generalized complete intersection (GCI) of affine schemes is introduced. The proofs of the following theorems are then sketched.

THeOREM 1. Suppose $X$ is a GCI of affine Cohen-Macaulay schemes of codimension $\leqslant 2$. Then if the versal deformation space of $X$ exists, it is smooth.

Theorem 2. Suppose $X$ is a GCI of determinantal schemes. Then if $\operatorname{dim} X$ is sufficiently low, $X$ is smoothable. In particular, if $X$ is as in Theorem 1 and $\operatorname{dim} X<4$ then $X$ is smoothable.
\end{abstract}

Introduction. In this note we introduce the concept of a generalized complete intersection (GCI), that is, an intersection of schemes all of whose subintersections are of generic height. We then announce the result that (Theorem 1) the parameter space of the versal deformation space of a GCI of hypersurfaces and Cohen-Macaulay (C-M) schemes of codimension 2 is smooth. We also obtain (Theorem 2) that in sufficiently low dimension a GCI of determinantal schemes is smoothable, in particular if $X$ is a GCI as in Theorem 1 and $\operatorname{dim} X<4$ then $X$ is smoothable. This generalizes results of ([Schaps], [Laksov]).

1. Definitions. Let $P=k\left[z_{1}, \ldots, z_{q}\right]$ be a polynomial ring in $q$ variables over a fixed field $k$. We call an ideal $J$ in $P$ Cohen-Macaulay if $P / J$ is a C-M ring of pure dimension.

Definition 1. Let $J_{1}, \ldots, J_{r}$ be ideals in $P$ and suppose for each subset $i_{1}, \ldots, i_{s}$ of $\{1, \ldots, r\}$ the ideal $J_{i_{1}}+\cdots+J_{i_{s}}$ is of height ht $J_{i_{1}}+\cdots+$ ht $J_{i_{s}}$. Then we call $J=J_{1}+\cdots+J_{r}$ a generalized complete intersection ideal (GCI).

We will see that (Lemma 1) if $J_{1}, \ldots, J_{r}$ are each complete intersections or C-M of codimension 2 , and $J$ is a GCI ideal, then $J_{1}, \ldots, J_{r}$ is a prime sequence in the following sense.

Definition 2. A sequence of ideals $J_{1}, \ldots, J_{r}$ in $P$ is a prime sequence iff $J_{i} \cap\left(J_{1}+\cdots+J_{i-1}\right)=J_{i}\left(J_{1}+\cdots+J_{i-1}\right)$ for $i=2, \ldots, r$.

We note that if the $J_{i}$ are of ht 1 our definitions correspond to the standard definitions of a complete intersection ideal. We also note if the $J_{i}$ are C-M then so is $J$.

Received by the editors July 14, 1975.

AMS (MOS) subject classifications (1970). Primary 14D15, $14 \mathrm{~B} 05$.

Copyright is 1977, American Mathematical Society 
2. Preliminary lemmas. Let $J_{1}, J_{2}$ be ideals in $P$ such that $J_{1}$ and $J_{2}$ are Cohen-Macaulay and $J_{1}+J_{2}$ is unmixed and ht $\left(J_{1}+J_{2}\right)=\mathrm{ht} J_{1}+$ ht $J_{2}$. If $J_{1}$ is a hypersurface or ht $J_{1}=2$ then

$$
J_{1} \cap J_{2}=J_{1} \cdot J_{2} \text {. }
$$

Proof. Clearly $J_{1} \cap J_{2} \supset J_{1} \cdot J_{2}$ so it suffices to show that $J_{1} \cap J_{2} \subset J_{1}$ $\cdot J_{2}$.

Suppose $J_{1}$ is generated by $\left(f_{1}, \ldots, f_{n}\right)$. Let $d=\sum a_{i} f_{i} \in J_{1} \cap J_{2}$ with $a_{i}$ $\in P$. If ht $J_{1}=1$ then $f_{1}$ is not a zero divisor in $P / J_{2}$, and thus $a_{1} \in J_{2}$ and so therefore $d \in J_{1} J_{2}$. So suppose ht $J_{1}=2$ and thus by [Schaps] we may assume that $f_{1}, \ldots, f_{n}$ are the minors of an $n \times(n-1)$ relation matrix $R=\left(r_{i j}\right)$ with columns $r_{1}, \ldots, r_{n-1}$. Then $d=\operatorname{det}\left[a, r_{1}, \ldots, r_{n-1}\right]$ where ${ }^{t} a=\left(a_{1}, \ldots, a_{n}\right)$. Expanding $d$ along its $n$th row we obtain $d=f_{n} a_{n}+b_{1} r_{n 1}+\cdots+b_{n-1} r_{n, n-1}$ $\in J_{2}$ for appropriate $b_{i}$. Thus $f_{n} a+\sum b_{i} r_{i} \in J_{2} P^{n}$. Again, multiplying by appropriate minors gives us $b_{j} J_{1} \subset\left(f_{n}, J_{2}\right)$ for all $j$, and since $J_{1}$ is not contained in any of the associated primes of $\left(f_{n}, J_{2}\right)$ we find $b_{j} \in\left(f_{n}, J_{2}\right)$. Thus $f_{n}\left(a+b_{j}^{\prime} r_{j}\right) \in J_{2} P^{n}$ and since $f_{n}$ can be assumed to be prime to $J_{2}$ we obtain $a+\sum b_{j}^{\prime} j_{j} \in J_{2} P^{n}$, so $d \in J_{1} J_{2}$.

Lemma 2. Let $J=J_{1}+J_{2}, B=P / J$ and $B_{i}=P / J_{i}$. If $J_{1} \cap J_{2}=J_{1} \cdot J_{2}$, then for any B-module,

$$
M \operatorname{Hom}_{B}\left(J / J^{2}, M\right) \simeq \operatorname{Hom}_{B_{1}}\left(J_{1} / J_{1}^{2}, M\right) \oplus \operatorname{Hom}_{B_{2}}\left(J_{2} / J_{2}^{2}, M\right) .
$$

Proof. Use $J / J^{2} \stackrel{\sim}{\longrightarrow} J_{1} / J_{1} J \oplus J_{2} / J_{2} J \simeq\left(J_{1} / J_{1}^{2} \otimes J_{2} / J_{2}^{2}\right) \otimes B$.

LEMMa 3. Under the hypothesis of Lemma 1

$$
\operatorname{Hom}_{B_{1}}\left(J_{1} / J_{1}^{2}, B\right) \stackrel{\sim}{\longrightarrow} \operatorname{Hom}_{B_{1}}\left(J_{1} / J_{1}^{2}, B_{1}\right) \otimes_{B_{1}} B
$$

Proof. We use techniques similar to those used in the proof of Lemma 1.

Lemma 4. Suppose $J_{1}, J_{2}$ are ideals such that $J_{1} \cap J_{2}=J_{1} J_{2}$. Then any flat deformation of $J_{1}$ and $J_{2}$ over an Artin or complete local ring $R$ lifts to a flat deformation of $J=J_{1}+J_{2}$.

Proof. A deformation over $R$ is flat iff all the relations can be lifted. The relations on a generating set of $J_{1}+J_{2}$ are generated by relations on $J_{1}$, relations on $J_{2}$ and trivial relations, all of which are liftable.

\section{Main theorems.}

THEOREM 1. Suppose $J_{1}, \ldots, J_{r}$ are either hypersurfaces or $C$ - $M$ of codimension 2 and $J=J_{1}+\cdots+J_{r}$ is a generalized complete intersection ideal. Then if the versal deformation space of $X=\operatorname{Spec}(P / J)$ exists, it has a smooth parameter space.

Proof. Let $I_{t}=J_{1}+\cdots+J_{t}, B_{t}=P / J_{t}, C_{t}=P / I_{t}$ and $B=P / J$. Then by Lemma $1, J_{t} \cap I_{t-1}=J_{t} \cdot I_{t-1}$, and by Lemma 2, 


$$
\operatorname{Hom}_{B}\left(J / J^{2}, B\right) \simeq \bigoplus_{t=1}^{r} \operatorname{Hom}_{B_{t}}\left(J_{t} / J_{t}^{2}, B\right)
$$

By Lemmas 1 and 3 we get

$$
\operatorname{Hom}_{B_{t}}\left(J_{t} / J_{t}^{2}, B\right) \simeq \operatorname{Hom}_{B_{t}}\left(J_{t} / J_{t}^{2}, B_{t}\right) \oplus_{B_{t}} B
$$

Thus $\operatorname{Hom}_{B}\left(J / J^{2}, B\right)$ is generated by the generators of the various $\operatorname{Hom}_{B_{t}}\left(J_{t} / J_{t}^{2}, B_{t}\right)$. Now (by [Schlessinger]), $X$ has a versal deformation space iff

$$
t_{B}=\operatorname{Hom}_{B}\left(J / J^{2}, B\right) / \operatorname{Im}\left(\operatorname{Hom}_{B}\left(\Omega_{P / k} \otimes B, B\right)\right)
$$

is finite dimensional as a $k$-vector space (where $\Omega_{P / k}$ and the map

$$
\operatorname{Hom}_{B}\left(\Omega_{P / k} \otimes B, B\right) \rightarrow \operatorname{Hom}\left(J / J^{2}, B\right)
$$

are as in [Schlessinger]).

We can take a $k$-basis $x_{1}, \ldots, x_{N}$ for $t_{B}$ consisting of multiples of the generators over $B$ of the various $\operatorname{Hom}_{B_{t}}\left(J_{t} / J_{t}{ }^{2}, B\right)$. Letting $T_{1}, \ldots, T_{N}$ be indeterminates, the generic first order deformation over $k[T] /(T)^{2}$ induces deformations of each of the $J_{t}$, each of which has a flat lifting over $k[[T]]$. Therefore, by Lemma 4, the deformation of $J$ can be lifted over $k[[T]]$, giving the versal deformation space, with smooth parameter space Spec[[T]].

Using techniques of [Laksov] we obtain

Theorem 2. Suppose $P=k\left[z_{1}, \ldots, z_{q}\right]$ with $k$ a fixed field.

Let $X_{1}, \ldots, X_{m}$ be determinantal schemes with $X_{t}=\operatorname{Spec}\left(P / J_{t}\right)$ and $J_{t}$ generated by the $l_{t} \times l_{t}$ minors of an $m_{t} \times n_{t}$ matrix. Put

$$
X=\operatorname{Spec}\left(P / J_{1}+\cdots+J_{m}\right)
$$

and suppose that $X$ has pure codimension equal to $\Sigma \operatorname{codim}\left(X_{t}\right)$.

Then if $X$ is a generalized complete intersection and if

$$
\operatorname{dim} X<\min _{t \in N}\left(m_{t}+n_{t}-2 l_{t}+3\right)
$$

where $N=\left\{t \mid X_{t}\right.$ is not a complete intersection $\}$ then $X$ is smoothable.

COROllary. Suppose each $X_{i}$ above is either a complete intersection or $C-M$ of codimension 2. Then $\operatorname{dim} X<4$ implies $X$ is smoothable.

\section{REFERENCES}

D. Laksov, Deformation of determinantal varieties, Univ. of Oslo (to appear).

M. Schaps, Deformations of Cohen-Macaulay schemes of codimension 2 and non-singular deformations of space curves (to appear). 
M. Schlessinger, Functors of Artin rings, Trans. Amer. Math. Soc. 130 (1968), 208-222. MR 36 \# 184.

Weizmann Institute of Science, P.O.B. 26, Rehovot, IsRael

Mathematical Sciences Department, Tel-Aviv University, Tel-Aviv, Israel (Current address of M. Schaps)

Current address (R. Mandelbaum): School of Mathematics, Institute for Advanced Study, Princeton, New Jersey 08540 